\title{
Patrones fenológicos de doce especies arbóreas del bosque montano de la Cordillera de Talamanca, Costa Rica
}

\author{
Marlen Camacho y Lorena Orozco \\ 1 Proyecto Silvicultura Bosques Naturales, CATE, Turrialba, Costa Rica. \\ Fax (507) 556 1533, Correo electrónico mcamacho@catie.ac.cr>, <lorozco@catie.ac.cr
}

Recibido 27-XI-1997. Corregido 11-V-1998. Aceptado 8-VI-1998.

\begin{abstract}
The phenology of 160 individuals from 12 tree species of Costa Rica's montane forests were observed monthly during 49 months, in order to evaluate the following phenophases: flowering, fructification and leafing (budding and falling). Different occurrence patterns were found for each event. Thus, flowering occurred continuously for one species but sub-annually for another. Four species showed annual flowering and the other six supra-annual flowering. Flowering was concentrated from November to May, at the end of the rainy season, and during the dry season. Fructification was continuous for three species, annual for two, and supra-annual for the other seven. There was more fruits production at the end of the dry season and during the first months of the rainy season. New leaf production was a very dynamic event and highly synchronic for all evaluated species, showing annual rhythms for six of them. Leaf falling was a continuous event throughout the year, without abrupt changes for all species under evaluation.
\end{abstract}

Key words: Cleyera, Drimys, Magnolia, montane tropical forest, Ocotea, phenological patterns, Quercus, Schefflera, Styrax, Vaccinium, Weinmannia.

El conocimiento y la comprensión de los patrones fenológicos de especies arbóreas en ecosistemas naturales son de interés básico en estudios ecológicos sobre biodiversidad, productividad y organización de las comunidades y de las interacciones de las plantas con la fauna; además, reviste gran importancia en programas de conservación de recursos genéticos, manejo forestal y planificación de áreas silvestres (Mooney et al. 1980, Huxley 1983).

En Costa Rica, los estudios fenológicos a nivel de especies o familias de interés forestal se han concentrado en los bosques secos y húmedos de las tierras bajas (Gentry 1974, Opler et al. 1975, Opler et al. 1980, Borchert 1980,
Bullock y Bawa 1981, Haber y Frankie 1982, Reich y Borchert 1982, Bullock et al. 1983, Marquis 1988, Fleming y Williams 1990, Céspedes 1991, Borchert 1995, Vílchez y Murillo 1995, Gómez y Fournier 1996, Madrigal 1997).

Los bosques montanos de Costa Rica, llamados comúnmente robledales de altura, conforman una masa boscosa de alrededor de 200000 ha, localizada principalmente en la Cordillera de Talamanca, entre 2000 y $3000 \mathrm{msnm}$. Son comunidades siempreverdes, florísticamente muy homogéneas y dominadas por una o varias especies del género Quercus, con representación de más del $70 \%$ del área basal y del $35 \%$ de la abundancia para el conjunto diamétrico 
mayor o igual a $10 \mathrm{~cm}$ (Jiménez et al. 1988, Blaser y Camacho 1991, Orozco 1992, Kappelle 1996, Orozco y Camacho 1997). A pesar de la importancia que tiene la conservación de estos ecosistemas naturales -no solo para salvaguardar su biodiversidad, sino también por la cantidad de servicios ambientales que prestan a la sociedad- son pocos los estudios realizados para profundizar en el entendimiento de los procesos dinámicos que aquí ocurren. En el campo de la fenología, se conocen solamente tres estudios publicados sobre especies de estos bosques (Céspedes 1991, Vílchez y Murillo 1995, Madrigal 1997). En este contexto, este estudio busca aportar información pionera acerca del comportamiento fenológico de doce especies arbóreas de los bosques montanos del sector noroccidental de la Cordillera de Talamanca.

\section{MATERIALES Y METODOS}

El estudio se realizó en cuatro sitios ubicados en un transecto altitudinal a lo largo de la Carretera Interamericana Sur, en el sector noroccidental de la Cordillera de Talamanca, Costa Rica: División (2 $050 \mathrm{msnm}$ ), Macho Mora (2 $550 \mathrm{msnm}$ ), Villa Mills (2 $700 \mathrm{msnm}$ ) y Asunción (2 $900 \mathrm{msnm}$ ), con una separación máxima de $20 \mathrm{~km}$ entre los sitios de menor y mayor altitud. El área que cubre los cuatro sitios se localiza entre los coordenadas geográficas 09'30'54" y 09³5'40" latitud norte y $83^{\circ} 43^{\prime} 05^{\prime \prime}$ y $83^{\circ} 44^{\prime} 50^{\prime \prime}$ longitud oeste. Una descripción detallada de estos sitios puede encontrarse en Orozco (1992) y Orozco y Camacho (1997).

Según registros de estaciones pluviográficas cercanas, la precipitación es de $3767 \mathrm{~mm}$ anuales cerca del sitio de menor altitud (2 260 msnm, Estación División) y de $2611 \mathrm{~mm}$ anuales en el sitio de mayor altitud (3 $000 \mathrm{msnm}$, Estación Villa Mills). En ambos casos existe una época seca bien definida entre diciembre a marzo-abril, con precipitaciones mensuales menores a $100 \mathrm{~mm}$ (Fig. 1).

Para la Estación Villa Mills (3 $000 \mathrm{msnm}$ ), la temperatura promedio anual es de $7.3^{\circ} \mathrm{C}$. Enero es el más frío, con temperaturas que bajan hasta $3^{\circ} \mathrm{C}$. La humedad relativa del aire es siempre muy alta, con promedios mensuales entre 88 y $96 \%$. El brillo solar en el mes con más sol (febrero) es de 7.5 horas/día en promedio y en el mes con menos sol (setiembre) es de 3 horas/día(Blaser y Camacho 1991).

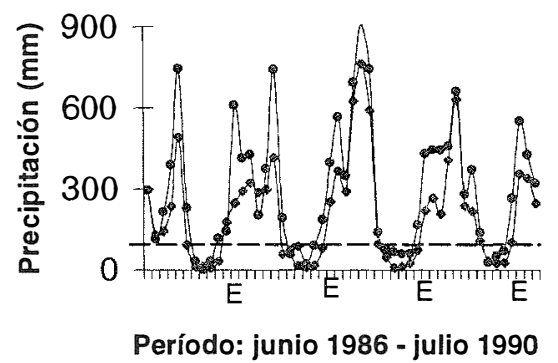

Fig. 1. Precipitación mensual total de las Estaciones División (2 260 msnm, - -) y Villa Mills (3 $000 \mathrm{msnm},-\not E$ ), cercanas a los sitios de estudio. La línea punteada representa el nivel de los $100 \mathrm{~mm}$ de precipitación mensual. La letra " $E$ " representa el primer mes de cada año.

De acuerdo con la clasificación de zonas de vida de Holdridge (1982), el sitio División pertenece al Bosque Pluvial Montano Bajo, y los restantes al Bosque Pluvial Montano. La topografía es plana en Villa Mills (0-15\%), ondulada en Asunción (20-40\%) hasta escarpada en División y Macho Mora (10-70\%). Según los análisis efectuados, los suelos son de muy baja fertilidad natural, fuertemente ácidos, con $\mathrm{pH}$ medidos en agua entre 3.5 y 4.6 , de textura franco-arcillosa, profundos y con baja pedregosidad (Jiménez et al. 1988, Blaser y Camacho 1991, Orozco 1992).

Recolección de datos: Las observaciones se realizaron en una red de parcelas temporales instaladas para el estudio de la estructura y composición florísticas de las comunidades boscosas del piso montano de Costa Rica (Orozco 1992). Se observaron 295 árboles de 34 especies arbóreas, de los cuales se evaluaron únicamente 160 árboles de 12 especies seleccionadas por su valor económico actual o potencial. Por diferencias en la composición florística de los rodales, tres de estas especies fueron muestreadas en los cuatro sitios, dos en tres sitios y siete especies en dos sitios (Cuadro 1). 


\section{CUADRO 1}

Especies observadas por sitio y parámetros dasométricos

\begin{tabular}{|c|c|c|c|c|c|c|c|c|c|}
\hline Especies & $\mathrm{D}$ & M & $\begin{array}{c}\text { Sitios } \\
\mathrm{V}\end{array}$ & A & Tot & $\begin{array}{l}\text { Dap } \\
(\mathrm{cm})\end{array}$ & $\begin{array}{l}\text { Alto } \\
\text { (m) }\end{array}$ & $\begin{array}{l}\text { D. Copa } \\
\text { (m) }\end{array}$ & $\begin{array}{l}\text { Est. } \\
\text { mad }\end{array}$ \\
\hline \multicolumn{10}{|l|}{ Araliaceae } \\
\hline Schefflera rodriguesiana & & & 7 & 4 & 11 & 36 & 20 & 15 & $S$ \\
\hline \multicolumn{10}{|l|}{ Cunoniaceae } \\
\hline Weinmannia pinnata & & & 8 & 5 & 13 & 33 & 23 & 11 & S \\
\hline \multicolumn{10}{|l|}{ Ericaceae } \\
\hline Vaccinium consanguineum & & 5 & 5 & 5 & 15 & 26 & 12 & 12 & I \\
\hline \multicolumn{10}{|l|}{ Fagaceae } \\
\hline Quercus seemannii & 4 & 5 & & & 9 & 55 & 32 & 21 & $\mathrm{D}$ \\
\hline Quercus copeyensis & 6 & 6 & 7 & 3 & 22 & 73 & 35 & 19 & $\mathrm{D}$ \\
\hline Quercus costaricensis & & & 12 & 4 & 16 & 67 & 33 & 22 & $\mathrm{D}$ \\
\hline \multicolumn{10}{|l|}{ Lauraceae } \\
\hline Ocotea pittierii & & & 5 & 5 & 10 & 25 & 17 & 8 & $S$ \\
\hline Ocotea austinii & & & 5 & 5 & 10 & 29 & 23 & 11 & S \\
\hline \multicolumn{10}{|l|}{ Magnoliaceae } \\
\hline Magnolia sororum & 5 & 5 & & & 10 & 31 & 24 & 11 & $S$ \\
\hline \multicolumn{10}{|l|}{ Styracaceae } \\
\hline Styrax argenteus & 5 & & 5 & & 10 & 24 & 20 & 10 & $S$ \\
\hline \multicolumn{10}{|l|}{ Theaceae } \\
\hline Cleyera theioides & 2 & 5 & 5 & 4 & 16 & 25 & 20 & 11 & $\mathrm{~S}$ \\
\hline \multicolumn{9}{|l|}{ Winteraceae } & \\
\hline Drimys granadensis & 3 & 5 & 5 & 5 & 18 & 33 & 19 & 13 & $\mathrm{~S}$ \\
\hline
\end{tabular}

Sitios: D=División (2 $050 \mathrm{msnm}$ ), M= Macho Mora (2 $550 \mathrm{msnm}$ ), V= Villa Mills (2 $700 \mathrm{msnm}$ ), A= Asunción (2 $900 \mathrm{msnm}$ ). Dap: diámetro a la altura del pecho promedio. Alto: altura total promedio. D. copa: diámetro de copa. Est. mad.: estrato de madurez: D: dosel superior (más de $28 \mathrm{~m}$ ), S: subdosel (entre 16 y 28), I: dosel inferior (menos de $16 \mathrm{~m}$ ) (adaptado de Orozco 1992).

El período de observación se extendió por 49 meses, de julio de 1986 a julio de 1990 , con un registro por mes calendario, durante los cuales se observaron los eventos de brotadura y caída del follaje, floración (incluyó brotes y flores maduras) y fructificación (incluyó frutos inmaduros y maduros). Se utilizó una simplificación de la escala de Fournier (1974) para cuantificar visualmente la magnitud de la producción de cada fenofase. La escala empleada va de 0 a 3 , donde " 0 " corresponde a una producción nula, "1" a una producción que cubre hasta un 33\% de la cobertura de la copa, "2" a una producción que cubre entre un 34 y un $66 \%$ de la cobertura de la copa y " 3 " a una producción que cubre más de un $67 \%$ de la cobertura de la copa.

La identificación de las especies fue realizada en el Herbario Nacional de Costa Rica. Para cada individuo observado se tomaron dos muestras testigo de herbario, que fueron depositadas en el Herbario Nacional y en el Herbario de la Unidad de Manejo de Bosques Naturales del CATIE.

Análisis de datos: Los registros mensuales de producción fueron convertidos a porcentajes utilizando el punto medio de cada clase. Para cada fenofase, se calcularon valores mensuales de producción por especie (PM) y de sincronía por especie (SM):

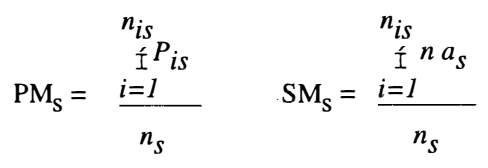

donde $\mathrm{s}=$ especie; $\mathrm{P}$ is = punto medio del valor de producción observado en el individuo i de la especie $\mathrm{s} ; \mathrm{n}_{\mathrm{s}}=$ número de individuos muestrados de la especie $\mathrm{s} ; \mathrm{na}_{\mathrm{s}}=$ número de individuos de la especie s en una determinada fenofase. 
Los promedios de los valores de producción de cada período de actividad se usaron para dar una indicación del tamaño de la cosecha. Un promedio menor a $20 \%$ indicó baja producción, entre 20 y $50 \%$ se consideró de mediana producción y de más de $50 \%$ de alta producción.

Además, se calculó un índice de sincronismo (IS) para determinar si un evento se manifestó de manera sincrónica, el cual resultó ser la mediana de los valores mensuales de sincronía $\left(\mathrm{SM}_{\mathrm{S}}\right)$ cuando la fenofase se mostró activa. Si el valor del índice fue mayor a 0.75 se consideró el evento como altamente sincrónico; entre 0.75 y 0.5 se consideró como sincrónico; entre 0.5 y 0.25 como poco sincrónico y menor a 0.25 como asincrónico.

Para examinar el efecto del sitio y del tiempo sobre la ocurrencia y la producción de cada fenofase se realizaron análisis de varianza para mediciones repetidas. Debido a que el postulado de la homogeneidad de las varianzas no se cumplió, los datos originales de producción promedio mensual fueron transformados (transformación angular) para utilizarlos en este análisis en particular. Se consideró que existió efecto de un factor solo cuando los resultados indicaron diferencias significativas a nivel de $P<0.05$.
Finalmente, se realizaron pruebas de correlación simple para establecer el grado de asociación entre el evento observado y la precipitación registrada en la estación pluviográfica de Villa Mills (3 $000 \mathrm{msnm}$ ).

\section{RESULTADOS}

Para las cuatro fenofases evaluadas, los análisis de varianza indican diferencias altamente significativas entre meses y entre especies, así como en la interacción sitios-meses y especiesmeses (Cuadro 2). Estos resultados sugieren que no existe un único patrón de ocurrencia del evento analizado en el ámbito de comunidad y que, por otra parte, ninguna de las especies contribuye a establecer picos de ocurrencia del evento en el tiempo. Aunque las diferencias por efecto del sitio son significativas en los eventos de floración y fructificación (Cuadro 2), un análisis de varianza realizado por especie revela que el sitio tiene influencia directa en la intensidad y la ocurrencia del evento solo en Quercus copeyensis $(P=0.0002$ en la floración y $P=0.0085$ en la fructificación).

\section{CUADRO 2}

Resultados del análisis de varianza para mediciones repetidas

\begin{tabular}{lcccccccc}
\multicolumn{1}{c}{ Efectos } & \multicolumn{2}{c}{ Floración } & \multicolumn{2}{c}{ Fructificación } & \multicolumn{2}{c}{ Brotadura } & \multicolumn{2}{c}{ Caída de hojas } \\
& $F$ & $P$ & $F$ & $P$ & $F$ & $P$ & $F$ & $P$ \\
Sitios & & & & & & & & \\
Especies & 3.09 & 0.0490 & 3.73 & 0.0127 & 1.27 & 0.2857 & 1.01 & 0.3887 \\
Meses & 9.53 & 0.0001 & 42.46 & 0.0001 & 9.19 & 0.0001 & 2.24 & 0.0001 \\
Meses-sitios & 12.00 & 0.0001 & 8.76 & 0.0001 & 24.68 & 0.0001 & 11.17 & 0.0001 \\
Meses-especies & 3.10 & 0.0001 & 3.32 & 0.0001 & 3.48 & 0.0001 & 2.53 & 0.0001 \\
& 5.87 & 0.0001 & 6.76 & 0.0001 & 4.63 & 0.0001 & 3.01 & 0.0001
\end{tabular}

$F$ : valor $\mathrm{F}$ en el análisis de varianza, $P$ : probabilidad.

El comportamiento fenológico por especie se describió con base en la evaluación de las variables de producción, sincronía, duración y la identificación de los patrones de foliación, floración y fructificación, siguiendo la clasificación propuesta por Newstrom et al. (1994).
Floración: Los patrones de floración (Fig. 2) muestra que Schefflera rodriguesiana presentó floración continua, con actividad sincrónica durante once meses de cada año y con cosechas de baja a mediana producción. Cleyera theioides mostró un patrón subanual, 
con cosechas asincrónicas y de baja producción, que en conjunto se extendieron por diez meses del año.

Cuatro especies mostraron floraciones anuales (Drimys granadensis, Weinmannia pinnata, Vaccinium consanguineum y Styrax argenteus), con una duración promedio de seis meses y con poca sincronía intraespecífica (IS entre 0.32 y 0.45). En todas estas especies, el evento mostró picos de cosechas mediana a altamente productivas, intercaladas con cosechas de baja producción cada dos años. Además, se dio una correlación significativa entre la precipitación y la ocurrencia de este evento en las cuatro especies: $W$. pinnata concentra la floración en la época de menor precipitación ( $\mathrm{r}=0.58, P=0.0001$, Spearman), mientras que las otras tres en la de mayor precipitación ( $\mathrm{r}=0.42, P=0.0023 ; \mathrm{r}=0.29, P=0.010$, $\mathrm{r}=0.45, P=0.0010$, Spearman, respectivamente).

Las seis especies restantes parecen tener patrones supranuales de floración. Ocotea pittieri mostró una floración cada dos años, que se inició en la época seca y se extendió por siete meses y $O$. austinii presentó un solo período de floración en cuatro años, que se extendió por trece meses. En ambas especies las cosechas fueron de bajas a medianamente productivas y poco sincrónicas (IS de 0.42 y 0.25 , respectivamente). Magnolia sororum mostró pequeñas manifestaciones del evento cada dos años, que se presentaron de manera asincrónica en los primeros dos o tres meses de la época lluviosa.

En las tres especies del género Quercus, el período de floración masculina fue más corto que el de la femenina y se manifestó con uno o dos meses de anticipación. En $Q$. copeyensis se presentó un prolongado período de floración femenina de doce meses de duración (en 1988), con pequeños picos de floraciones masculinas de corta duración (dos a tres meses) y asincrónicos, entre noviembre y marzo de cada año. En $Q$. seemannii ocurrió igualmente un período de floración femenina entre setiembre de 1987 y marzo de 1988, con picos anuales de floración masculina, entre noviembre-diciembre y enero, excepto en 1990. Q. costaricensis presentó un evento más sincrónico y compacto, con una floración femenina de diez meses que inició durante la estación lluviosa y presentó un pico de mayor producción en la época seca y una sola floración masculina de siete meses, con un pico de mayor producción de octubre a enero, al final de la época lluviosa e inicios de la seca. Estos resultados sugieren que algunas especies de Quercus de este sector de la Cordillera de Talamanca pueden presentar floraciones masculinas cada año, pero con verdaderos períodos reproductivos cada dos o más años.

Los patrones de floración antes mencionados exhiben una alta correlación con el tamaño de la semilla de las especies estudiadas $(r=0.730, P=$ 0.0001 , Spearman). Así, las especies de floración continua y subanual poseen semillas muy pequeñas (menos de $2 \mathrm{~mm}$ ); las especies con picos de floración anual bien definidos, semillas pequeñas (entre 2 y $5 \mathrm{~mm}$ ), y las especies con patrones de floración supranuales, semillas medianas a grandes (mayores a $8 \mathrm{~mm}$ y hasta más de $20 \mathrm{~mm}$ ).

Fructificación: La identificación de los patrones de fructificación se torna difícil en algunas especies, ya sea porque los frutos de la misma cosecha permanecen en el árbol por largo tiempo (caso de $D$. granadensis, $C$. theioides, $S$. argenteus y $M$. sororum); o por el contrario, desaparecen rápidamente de la copa del árbol porque los frutos son muy apetecibles para la fauna silvestre (como las Ocotea), o porque son abortados (como en los Quercus).

Según la Fig. 2, tres especies mostraron fructificación continua ( $S$. rodriguesiana, $C$. theioides y $D$. granadensis), pues el evento se manifestó durante todos los meses de observación, con alta sincronía entre los individuos y con cosechas muy productivas. Se presume que el patrón de $D$. granadensis es anual, pero no identificable debido a la persistencia de los frutos en el árbol.

Dos especies mostraron fructificación anual con tendencia a la supranualidad (W. pinnata y $V$. consanguineum). En W. pinnata, el evento se manifestó, de manera sincrónica, dos meses después de iniciada la floración y se extendió por seis meses de cada año, con cosechas de mediana a alta producción en picos supranuales de dos años. En $V$. consanguineum, la fructificación se presentó tres meses después de iniciada la floración y se extendió por cuatro a seis meses, con incrementos de cosechas cada dos años. 


\section{Floración}

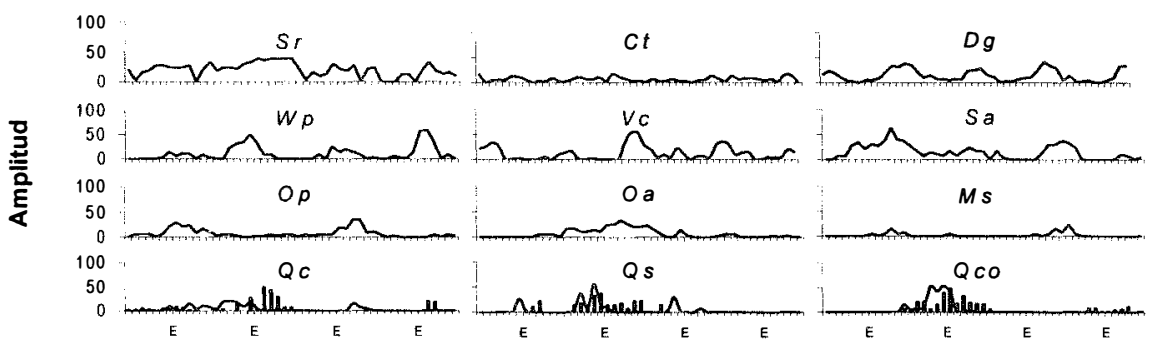

\section{Fructificación}
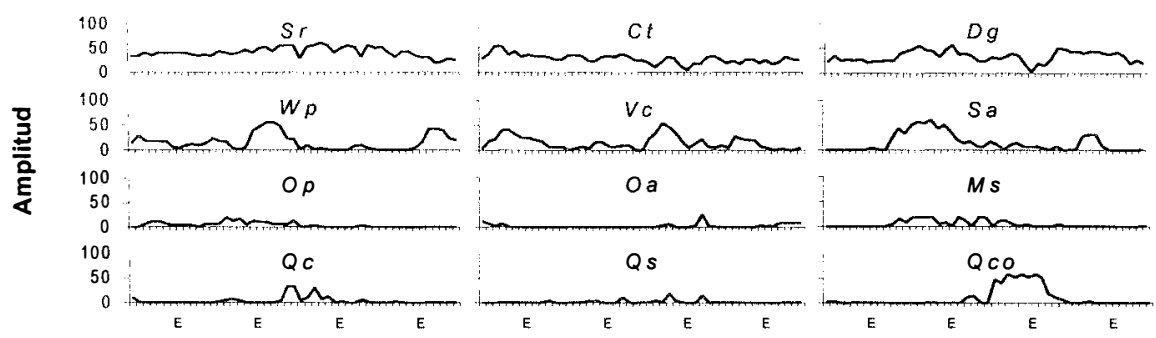

\section{Brotadura de hojas}
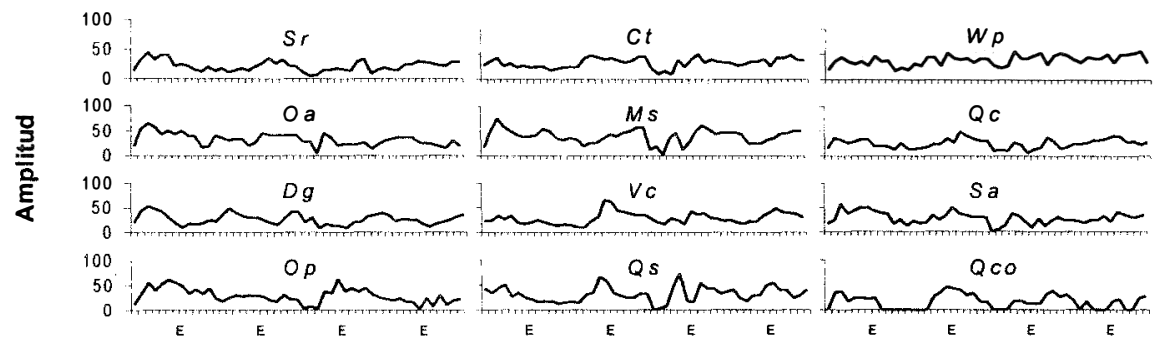

\section{Caída de hojas}

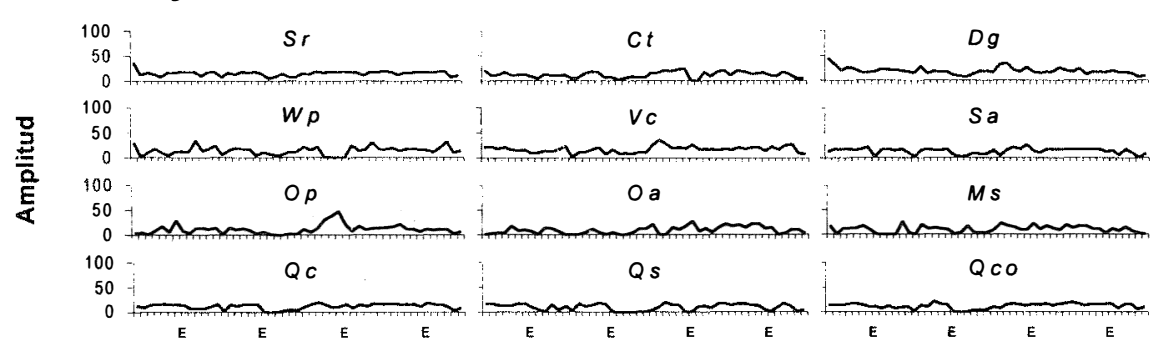

Fig. 2. Patrones fenológicos de las especies estudiadas. Floración: Continuo: $S r$, Subanual: $C t$; Anual: $D g, W p, V c$ y $S a$; Supranual: $O p, O a, M s, Q c, Q s$ y $Q c o$. Fructificación: Continuo: $S r, C t$ y $D g$; Anual: $W p$ y $V c$; Supranual: $S a, O p, O a, M s, Q c$, $Q s$ y $Q c o$. Brotadura de hojas: Continuo: $S r, C t, W p, O a, M s$ y $Q c$; Anual: $D g, V c, S a, O p, Q s$ y $Q c o$. Caída de hojas: Continuo: $S r$, Ct y Dg; Subanual: Wp, Vc, Sa, Op, Oa, Ms, Qc, Qs y Qco. Especies: Sr: Schefflera rodriguesiana; Ct:Cleyera theioides; Dg: Drimys granadensis, Wp: Weinmannia pinnata, Vc: Vaccinium consanguineum, Sa: Styrax argenteus; Op: Ocotea pittieri, Oa: O. austinii, Ms: Magnolia sororum, Qc: Quercus copeyensis, Qs: Q. seemanni y Qco:Q. costaricensis. En Quercus, las barras corresponden a la floración masculina y las líneas a la femenina. La letra "E" representa el primer mes de cada año. 
S. argenteus y $O$. pittieri presentaron picos supranuales de fructificación cada dos años. En la primera especie, estos picos fueron de muy baja producción, se presentaron cuatro a cinco meses después de iniciada la floración y perduraron por cinco o seis meses, durante la época lluviosa. La segunda especie presentó dos picos de fructificación: el primero altamente producuivo y sincrónico se inició cuatro meses después del primer pico de floración y se extendió por casi doce meses; el segundo dos años después, de poca producción, asincrónico y con una duración de solo tres meses.

En el resto de las especies se observó solo un período de fructificación en los cuatro años de observación. En $Q$. costaricensis se presentó una cosecha de frutos, sincrónica y muy productiva un año después de iniciada la floración femenina y seis meses después de finalizada la floración masculina, la cual se extendió por ocho meses (de agosto 88 a marzo 89).

M. sororum inició un período de fructificación una vez finalizada la floración, el cual se extendió por casi un año pues las infrutescencias persistieron en el árbol por largo tiempo, con una cosecha poco sincrónica (IS $=0.23$ ) y de baja producción.

$O$. austinii presentó una pequeña y asincrónica cosecha de frutos en la época de lluvias, ocho meses después del principal pico de floración, y lo que parece una nueva manifestación del evento al final del estudio, dos años después.

Q. copeyensis y $Q$. seemannii mostraron cosechas que se extendieron de mayo a diciembre en la primera y de julio a febrero en la segunda. Ambas cosechas pueden considerarse asincrónicas (IS $=0.19$ y 0.16 , respectivamente) y de baja a mediana producción.

Foliación: Las comunidades vegetales del piso Montano y Montano Bajo son típicamente perennifolias y no presentan especies arbóreas caducifolias o semicaducifolias.

La brotadura de las hojas es un evento muy dinámico, a juzgar por los resultados presentados en la Fig. 2. Se manifestó altamente sincrónico (IS mayor a 0.71) en todas las especies estudiadas, con picos de cosechas de mediana a alta producción. Además, mostró una asociación significativa con la precipitación mensual en seis de las especies estudiadas: $D$. granadensis, $V$. consanguineum, $S$. argenteus, O. pittieri, $Q$. seemannii y $Q$. costaricensis $(\mathrm{r}=0.40$, $P=0.0042, \quad \mathrm{r}=0.39, \quad P=0.0048 ; \quad \mathrm{r}=0.41$, $P=0.0031 ; \quad \mathrm{r}=0.40, \quad P=0.0036 ; \quad \mathrm{r}=0.34$, $P=0.0101 ; \mathrm{r}=0.37, P=0.0080$, Spearman), con picos anuales en los meses más secos.

La mayoría de las especies presentaron un descenso en la producción de hojas nuevas entre agosto y octubre de 1988, meses en que la precipitación alcanzó los mayores niveles en los cuatro años de estudio (más de $500 \mathrm{~mm}$ mensuales).

La caída de las hojas ocurrió de manera continua y sin cambios abruptos a través del año, en las doce especies estudiadas (Fig. 2). También aquí, el evento fue altamente sincrónico (IS mayor a 0.83 ), aunque de baja a mediana productividad. Cuatro especies mostraron correlación con la precipitación: S. argenteus, Q. costaricensis, D. granadensis y $Q$. seemannii $(\mathrm{r}=0.41$, $P=0.0031 ; \quad \mathrm{r}=0.40, \quad P=0.0039, \quad \mathrm{r}=0.38$, $P=0.0020 ; \mathrm{r}=0.45, P=0.0038$, Spearman, respectivamente). En las dos primeras, la caída de las hojas se intensificó en la época lluviosa, mientras que en las otras dos ocurrió en la época seca.

\section{DISCUSION}

Los resultados obtenidos de los análisis de varianza para mediciones repetidas son similares a otros estudios (Kinnaird 1992, Chin Sun et al. 1996). Las diferencias significativas entre especies revelan la variedad de patrones existentes producto de las diferentes fisiologías y las diferencias entre meses demuestran la variación de la producción en el tiempo. La ausencia de diferencias significativas entre sitios en la mayoría de las especies demuestra que estas tienen comportamientos fenológicos similares en un área determinada, restringida en este caso, a los bosques montanos del sector noroccidental de la Cordillera de Talamanca, Costa Rica. Como lo señalan Blaser y Camacho (1991) y Orozco (1992), estos 
bosques presentan parámetros estructurales muy similares, aunque con variaciones locales en composición florística. Por otra parte, los diagramas de precipitación de la Fig. 1, que abarcan estaciones localizadas muy cerca de los sitios a menor y mayor altitud, muestran la existencia de un único patrón de lluvias en la zona.

La actividad foliar sugiere una cierta estacionalidad a nivel de la comunidad, con picos de brotadura del follaje al final de la época lluviosa para los Quercus y durante la época seca para la mayoría de las otras especies estudiadas. Por su parte, la caída de las hojas, aunque con un patrón continuo, parece intensificarse durante los últimos meses de la época de lluvias. Este comportamiento ha sido señalado también por Céspedes (1991) para $Q$. seemannii y por otros autores en especies de diferentes tipos de bosques (Frankie et al. 1974, Fournier 1976, Borchert 1995).

El evento de floración de la mayoría de las especies estudiadas mostró picos hacia el final de la época seca y durante la lluviosa, mientras que las floraciones supranuales de los Quercus presentaron un incremento de la cosecha al final de la época lluviosa e inicios de la seca. Al igual que en este estudio, Kaul et al. (1986) observaron floraciones de Quercus de los bosques montanos de Borneo al final de la época lluviosa. Los mismos autores señalan que como la polinización en este género es anemófila, no necesita que la floración coincida con los picos de poblaciones de insectos; y más aun, que probablemente no sea efectiva bajo condiciones de alta precipitación. Para algunas de las otras especies (Schefflera, Magnolia, Drimys) diversos autores (Hartshorn 1983, Lawton 1983, Rojas et al. 1992) coinciden en ubicar la floración durante los meses de lluvias, ya que insectos como abejas, escarabajos, mariposas y avispas son posibles agentes polinizadores.

Para las especies estudiadas, resulta evidente la concentración de las fructificaciones durante la época lluviosa. La manifestación asincrónica del evento en varias especies $(M$. sororum, O. austinni, y $Q$. seemannii) y los valores obtenidos en el análisis de varianza para $Q$. copeyensis $(P=0.0085)$ sugieren que estas especies pueden tener comportamientos diferentes o desfasados entre los cuatro sitios muestreados. En el caso de $M$. sororum, se comprobó que la especie muestra un patrón diferente en otros sitios: cosechas anuales simultáneas de flores y frutos, sumamente productivas y que persistían por seis o siete meses durante la época de lluvias en árboles que crecían en condiciones de completa apertura, lo cual lo confirma las observaciones de Rojas et al. (1992). Como lo señalan Newstrom et al. (1994) las variaciones de comportamiento pueden deberse a las condiciones de luz que caracterizan los diferentes ecosistemas.

Para Q. costaricensis, Blaser (1987) citado por Quirós (1990), informa de una cosecha masiva entre 1985 y 1986, que con relación a la cosecha de 1988-89 sugiere que la especie presenta periodos de fructificación altamente productivos cada tres años; comportamiento que Burger (1983) interpreta como una estrategia para saturar los herbívoros.

Los síndromes de dispersión de las diásporas de las especies estudiadas (según Burger 1977, Lawton 1983, Rojas et al. 1992 y observaciones personales) sugieren que se da un claro predominio de la zoocoria, a excepción posiblemente de $W$. pinnata, la cual posee frutos pequeños, secos, que se abren parcialmente por un lado, liberando las semillas (Gentry 1993). Considerando que la mayoría de las especies (excepto $Q$. costaricensis y las continuas $S$. rodriguesiana y $C$. theioides) concentran la producción de frutos en los primeros meses de la época lluviosa, se presume que a finales de la época seca e inicios de la lluviosa las poblaciones de animales y aves se incrementan en los bosques montanos de la Cordillera de Talamanca, debido a la mayor disponibilidad de fuentes alimenticias (néctar, polen, frutos, insectos polinizadores). Esta aseveración apoya la impresión de Wolf (1976), quien al estudiar las poblaciones de aves del Cerro de la Muerte (2 $900 \mathrm{msnm}$ ), muy cerca a los sitios de estudio, observó una marcada coincidencia entre la fructificación y las épocas de procreación de la avifauna. 


\section{RESUMEN}

Durante 49 meses se observó la fenología de 160 árboles de 12 especies arbóreas de los bosques montanos de Costa Rica, mediante la evaluación de cuatro fenofases: floración, fructificación, brotadura y caída del follaje. Se encontraron diferentes patrones de ocurrencia en cada evento. La floración ocurre de manera continua en una especie y subanual en otra, cuatro especies muestran floraciones anuales y las seis restantes floraciones supranuales. De manera general, las floraciones se concentran de noviembre a mayo, a finales de la época lluviosa y durante la seca. La fructificación es continua en tres especies, anual en dos y supranual en las siete restantes, con mayor producción de frutos hacia el final de la época seca y primeros meses de la lluviosa. La brotadura de las hojas es un evento muy dinámico y altamente sincrónico en todas las especies estudiadas, con ritmos anuales en seis especies. La caída de las hojas ocurre de manera continua y sin cambios abruptos a través del año, en las doce especies estudiadas.

\section{AGRADECIMIENTOS}

A la Cooperación Suiza para el Desarrollo (COSUDE) por el financiamiento otorgado al Proyecto Silvicultura de Bosques Naturales del Centro Agronómico Tropical de Investigación y Enseñanza (CATIE), en el seno del cual se realizó este estudio. A E. Arnáez, Q. Jiménez y B. Vílchez y a tres revisores anónimos por sus comentarios para mejorar el manuscrito.

\section{REFERENCIAS}

Blaser, J. \& M. Camacho. 1991. Estructura, composición y aspectos silviculturales de un bosque de roble (Quercus spp.) del piso montano en Costa Rica. CATE, Turrialba, Costa Rica. 68p.

Borchert, R. 1980. Phenology and ecophysiology of tropical trees: Erythrina poeppigiana O.F. Cook. Ecology 61: 1065-1074.

Borchert, R. 1995. Phenology and flowering periodicity of neotropical dry forest species: evidence from herbarium collections. J. Trop. Ecol. 12: 65-80.

Bullock, S.H. \& K. Bawa. 1981. Sexual dimorphism and the annual flowering pattern in Jacaratia dolichaula (D. Smith) Woodson (Caricaceae) in a Costa Rican rain forest. Ecology 62: 1494-1504.
Bullock, S.H., J. H. Beach \& K. Bawa. 1983. Episodic flowering and sexual dimorphism in Guarea rhopalocarpa Radlk. (Meliaceae) in a Costa Rican rain forest. Ecology 64: 851-862.

Burger, W. 1977. Flora costaricensis: Fagaceae. Fieldiana Bot. 40: 74-75.

Burger, W. 1983. Quercus costaricensis, p. 318. In D. Janzen (ed.). Costa Rican Natural History. University of Chicago, Chicago.

Céspedes, R. 1991. Fenología de Quercus semannii Lieb. (Fagaceae) en Cartago, Costa Rica. Rev. Biol. Trop. 39: 243-248.

Chin Sun, B. A. K., K. A. Kristensen, V. Munyaligoga, J. Mvukiyumwami, K. Kajondo \& T. Moermond. 1996. Tree phenology in a tropical montane forest in Rwanda. Biotropica 28: 668-681.

Fleming, T. H. \& C. F. Williams. 1990. Phenology, seed dispersal and recruitment in Cecropia peltata (Moraceae) in Costa Rican tropical dry forest. J. Trop. Ecol. 6: 163178.

Fournier, L. A. 1974. Un método cuantitativo para la medición de carácterísticas fenológicas en árboles. Turrialba 24: 422-423.

Fournier, L. A. 1976. Observaciones fenológicas en el bosque húmedo premontano de San Pedro de Montes de Oca, Costa Rica. Turrialba 26: 54-59.

Frankie, G. W., H. B. Baker \& P. A. Opler. 1974. Comparative phenological studies of trees in tropical wet and dry forest in a lowlands of Costa Rica. J. Ecol. 62: 881-919.

Gentry, A. H. 1974. Flowering phenology and diversity in tropical Bignoniaceae. Biotropica 6: 64-68.

Gentry, A. H. 1993. Woody plants of Northwest South America (Colombia, Ecuador, Peru). Conservation International, Washington, DC. 895 p.

Gómez F., P. \& L. A. Fournier. 1996. Fenología y ecofisiología de dos poblaciones de Tabebuia rosea (roble sabana) en Costa Rica (Bignoniaceae). Rev. Biol. Trop. 44: $61-70$

Haber, W. A. \& G. W. Frankie. 1982. Pollination of Luehea (Tiliaceae) in CostaRican deciduous forest. Ecology 63: 1740-1750. 
Hartshorn, G. S. 1983. Drimys granadensis, p. 236. In D. Janzen (ed.). Costa Rican Natural History. The University of Chicago Press, Chicago.

Holdridge, L. R. 1982. Ecología basada en zonas de vida. IICA, San José, Costa Rica. 216 p.

Huxley, P. A. 1983. Phenology of tropical woody perennials and seasonal crop plants with reference to their management in agroforestry systems, p. 503-525. In P. A. Huxley, (ed.). Plant research and agroforestry. International Center for Research in Agroforestry, Nairobi, Kenia.

Jiménez, W., A. Chaverri, R. Miranda \& I. Rojas. 1988. Aproximaciones silviculturales al manejo de un robledal (Quercus spp.) en San Gerardo de Dota, Costa Rica. Turrialba 38: 208-214.

Kaul, R. B., E. C. Abbe \& L. B. Abbe. 1986. Reproductive phenology of the oak family (Fagaceae) in the lowland rain forest of Borneo. Biotropica 18: 51-55.

Kappelle, M: 1996. Los bosques de roble (Quercus) de la Cordillera de Talamanca, Costa Rica: biodiversidad, ecología, conservación y desarrollo. Universidad de Amsterdam, Amsterdam, Países Bajos/Instituto Nacional de Biodiversidad, Heredia, Costa Rica. 336 p.

Kinnaird, M. F. 1992. Phenology of flowering and fruiting of an East African riverine forest ecosystems. Biotropica 24: 187-194.

Lawton, R. 1983. Didymopanax pittieri, p. 233. In D. Janzen (ed.). Costa Rican Natural History. The University of Chicago Press, Chicago.

Madrigal Jiménez, T. A. 1997. Fenología y ecofisiología del Quercus oocarpa (Fagaceae) Cartago, Costa Rica. Rev. Biol. Trop. 44(3)/45(1): 117-123.

Marquis, R. J. 1988. Phenological variation in the neotropical understory shrub Piper arieianum: causes and consequenses. Ecology 69: 1552-1565.

Mooney, H. A., O. Bjorkman, A. E. Hall, E. Medina \& P. B. Tomlinson. 1980. The study of physiological ecology of tropical plants -current status and needs. Bio-Science 30: $22-26$.
Newstrom, L. E., G. W. Frankie \& H. G. Baker. 1994. A new classification for plant phenology based on flowering patterns in lowland tropical rain forest trees at $\mathrm{La}$ Selva, Costa Rica. Biotropica 26: 141-159.

Opler, P. A., H. G. Baker \& G. W. Frankie. 1975. Reproductive biology of some Costa Rican Cordia species (Boraginaceae). Biotropica 7: 234-240.

Opler, P. A., G. W. Frankie \& H. G. Baker. 1980 Comparative phenological studies of treelet and shrub species in tropical wet and dry forest in a lowlands of Costa Rica. J. Ecol. 68: 167-188.

Orozco, L. 1992. Estudio ecológico y de estructura horizontal de seis comunidades boscosas de la Cordillera de Talamanca, Costa Rica. CATE, Turrialba, Costa Rica. $33 \mathrm{p}$.

Orozco, L. \& M. Camacho. 1997. Cambios estructurales y florísticos en el bosque montano de la Cordillera de Talamanca, Costa Rica. Rev. For. Cent. 19: 32-36.

Quirós, L. 1990. Caracterización, almacenamiento y conservación de semillas de Quercus costaricensis Liebmann (encino). Tesis Lic., Universidad Nacional. Heredia, Costa Rica. 84 p.

Reich, P. B. \& R. Borchert. 1982. Phenology and ecophysiology of the tropical tree Tabebuia neochrysantha (Bignoniaceae). Ecology 63: 294-299.

'Rojas, F., G. Torres, E. Arnáez \& I. Moreira. 1992. Especies forestales tropicales. Magnolia. Editorial Tecnológica de Costa Rica, Cartago, Costa Rica. 6 p.

Vílchez, B. \& O. Murillo. 1995. Análisis fenológico y de la biología reproductiva del jaúl en Costa Rica. Tecnología en Marcha (Costa Rica) 12: 65-73.

Wolf, L. L. 1976. Avifauna of the Cerro de la Muerte region, Costa Rica. Am. M. Novitates 2606: 1-37. 